\title{
Assessing learning processes with the gain-loss model
}

\author{
Luca Stefanutti • Pasquale Anselmi • Egidio Robusto
}

Published online: 20 November 2010

(C) Psychonomic Society, Inc. 2010

\begin{abstract}
Within the framework of knowledge space theory, a probabilistic skill multimap model for assessing learning processes is proposed. The learning process of a student is modeled as a function of the interaction between his competence state and the effect of a learning object on specific skills. Model parameters are initial probabilities of the skills, effects of learning objects on gaining and losing the skills, careless error, and lucky guess probabilities of the problems. A simulation study assessed model identifiability and goodness-of-recovery under several conditions. Practical implications of using the model are discussed, and the MATLAB code for simulating, estimating and testing it is available in the Psychonomic Society supplemental archive.
\end{abstract}

Keywords Learning process $\cdot$ Learning object $\cdot$ Knowledge space $\cdot$ Skill map $\cdot$ Formative assessment

\section{Introduction}

Formative assessment evaluates specific knowledge, skills and abilities of students to guide teaching and learning at an individual level (DiBello \& Stout, 2007). It helps the teacher to determine if students have learned what they were supposed to, as well as ascertaining whether an educational intervention has been effective in promoting specific learning or not. Models for formative assessment have been proposed within the frameworks of knowledge assessment (see, e.g., Albert \& Held, 1999; Korossy, 1999;

L. Stefanutti $(\varangle) \cdot$ P. Anselmi $\cdot$ E. Robusto

Department of Applied Psychology, University of Padua,

via Venezia 8 ,

35131, Padua, Italy

e-mail: luca.stefanutti@unipd.it
Schrepp, 1999; Schrepp, Held, \& Albert, 1999) and cognitive diagnosis (see, e.g., Almond, DiBello, Moulder, \& Zapata-Rivera, 2007; Gierl, 2007; Roussos, Templin, \& Henson, 2007; Stout, 2007). They pinpoint students' specific strengths and weaknesses by defining multidimensional skill profiles.

Within the context of knowledge assessment, we propose the gain-loss model (GaLoM), a probabilistic model for assessing the knowledge of students in the different steps of the learning process, and the effectiveness of the educational intervention in promoting specific learning. In particular, the model enables the teacher to evaluate whether the didactic intervention is appropriate for maintaining skills that students already possess, and for promoting the learning of new specific skills.

The theoretical framework of the model is knowledge space theory (Doignon \& Falmagne, 1985) that, consistent with the aims of formative assessment, provides a non numerical, multidimensional representation of the characteristics of a person. In knowledge space theory, given a collection $Q$ of problems (or items) in a knowledge domain, the knowledge state of a person is the collection of all the problems that this person is capable of solving, and a knowledge structure is the collection of knowledge states that contains at least the empty set $\varnothing$ and the domain $Q$ (Doignon \& Falmagne, 1985, 1999; Falmagne, Doignon, Koppen, Villano, \& Johannesen, 1990; Lukas \& Albert, 1999). The GaLoM focuses on the specific skills that a person must possess in order to solve the problems. In the present formalization, given a collection $S$ of skills, the competence state of a person is the collection $C \subseteq S$ of non directly observable skills possessed by him, and which underlie his observable responses to the problems. A competence structure is then a pair $(S, C)$, where $C$ is a collection of subsets of $S$. The skills are elementary and 
indecomposable units that may represent either declarative or procedural knowledge, including notions, abilities, solution procedures, tricks, and so on. The focus on them makes the model particularly suitable for didactic practice, because it enables the teacher to theoretically explain the observed responses, and to predict responses on another collection of problems. Moreover, the teacher is helped in identifying which skills should be taught so that a previously unsolvable problem becomes solvable. Competence-based extensions of knowledge space theory for implementing personalized learning in technologyenhanced learning systems have been proposed by Conlan, Hockemeyer, Wade, and Albert (2002), Heller, Steiner, Hockemeyer, and Albert (2006) and Hockemeyer (2003). In a recent work, Heller and Repitsch (2008) described how skill functions representing assignments of skills to problems that come from different sources (e.g., different domain experts) can be consistently integrated into a global skill assignment.

The GaLoM models the learning process of the students as a function of the interaction between their competence state and the effect of an educational intervention, called a "learning object". With the term learning object we refer to every didactic intervention that supports learning and has the potential capability of changing the competence state of the students. Learning objects could be general courses on particular topics, instructional contents (including texts, web pages, images, sounds, videos), glossaries of terms, quizzes, exercises, case studies, educational games, and so on. The model assesses the effect of learning objects on the attainment of skills required to solve problems in a given field of knowledge. Via the competency model, a skill multimap (Doignon, 1994; Doignon \& Falmagne, 1999) associates, with each problem, a collection of subsets of skills that are necessary and sufficient to solve it.

The GaLoM is characterized by five types of parameters. The parameter concerning the initial probability of the skills specifies what skills the students possess before the teaching begins. The gain and loss parameters respectively specify if the students attain and eventually lose specific skills as a result of the learning object they have been presented with. The careless error parameter specifies if the students who master a problem fail it through inattention, whereas the lucky guess parameter specifies if the students who do not master a problem solve it by guessing.

In this article, we present the GaLoM along with a procedure for estimating its parameters and a simulation study of some of the properties of this model under several conditions. An empirical application of the model to real data is described in Robusto, Stefanutti, and Anselmi (2010).

The mathematical specification of the GaLoM and maximum likelihood estimation of its parameters are introduced in the following two sections. Next, a simulation study that assesses identifiability and goodness-of-recovery of the model is provided. Finally, practical implications of using the model are explored and discussed. The MATLAB code for simulating, estimating and testing the model is available in the Psychonomic Society supplemental archive.

\section{The gain-loss model}

The GaLoM assesses the effect of $m$ different learning objects in processes with two assessment steps. The experimental design is $t_{1} \rightarrow I_{o} \rightarrow t_{2}$, where $I$ represents the educational intervention carried out with learning object $o \in\{1,2, \ldots, m\}$, and $t_{1}$ and $t_{2}$ are the pretest and posttest, respectively.

Let $S$ be a finite and non empty set of discrete skills, and $C$ be any subset of $S$. Let $\mathbf{C}_{1}$ and $\mathbf{C}_{2}$ be two discrete random variables whose realizations are the competence states of a student at the pretest and posttest, respectively. Let $Q$ be a nonempty finite set containing $n$ dichotomous problems, and $\mathbf{R}_{1}$ and $\mathbf{R}_{2}$ be two discrete random variables whose realizations are the response patterns $\mathbf{r} \in\{0,1\}^{n}$ of a student at the pretest and posttest, respectively.

Assumptions of the model are:

1. The response patterns $\mathbf{R}_{1}$ and $\mathbf{R}_{2}$ are locally independent, given the states $\mathbf{C}_{1}$ and $\mathbf{C}_{2}: P\left(\mathbf{R}_{1}, \mathbf{R}_{2} \mid \mathbf{C}_{1}, \mathbf{C}_{2}\right)=$ $P\left(\mathbf{R}_{1} \mid \mathbf{C}_{1}\right) P\left(\mathbf{R}_{2} \mid \mathbf{C}_{2}\right)$

2. The initial state $\mathbf{C}_{1}$ does not depend on learning object $o$.

3. State $\mathbf{C}_{2}$ depends on previous state $\mathbf{C}_{1}$ and on learning object $o$.

It follows that the conditional probability that $\mathbf{r}_{1}$ and $\mathbf{r}_{2}$ are the response patterns of a randomly sampled student at the pretest and posttest, given learning object $o$, is:

$$
\begin{aligned}
& P\left(\mathbf{R}_{1}=\mathbf{r}_{1}, \mathbf{R}_{2}=\mathbf{r}_{2} \mid o\right)=\sum_{C \subseteq S} \sum_{D \subseteq S} P\left(\mathbf{R}_{1}=\mathbf{r}_{1} \mid \mathbf{C}_{1}=C\right) \\
& \quad \times P\left(\mathbf{R}_{2}=\mathbf{r}_{2} \mid \mathbf{C}_{2}=D\right) P\left(\mathbf{C}_{2}=D \mid \mathbf{C}_{1}=C, o\right) P\left(\mathbf{C}_{1}=C\right),
\end{aligned}
$$

where $P\left(\mathbf{C}_{1}=C\right)$ is the initial probability of the state $C$ at the pretest, $P\left(\mathbf{C}_{2}=D \mid \mathbf{C}_{1}=C, o\right)$ is the transition probability from state $C$ at the pretest to state $D$ at the posttest, $P\left(\mathbf{R}_{1}=\mathbf{r}_{1} \mid \mathbf{C}_{1}=C\right)$ and $P\left(\mathbf{R}_{2}=\mathbf{r}_{2} \mid \mathbf{C}_{2}=D\right)$ are the emission probabilities of response patterns $\mathbf{r}_{1}$ and $\mathbf{r}_{2}$ at the pretest and posttest, respectively.

Equation (1) is the basic equation of the model. Assuming total independence among the skills in $S$, the probability $P\left(\mathbf{C}_{1}=C\right)$ is resolved according to Eq. (2):

$$
P\left(\mathbf{C}_{1}=C\right)=\prod_{s \in S} \pi_{s}^{w(s, C)}\left(1-\pi_{s}\right)^{1-w(s, C)}
$$


where $\pi_{s}=P\left(s \in \mathbf{C}_{1}\right)$ is the probability that skill $s$ belongs to the initial competence state, and $w(s, C) \in\{0,1\}$ is equal to 1 if skill $s$ belongs to state $C$.

Given the dependence among the skills modelled at time $t_{1}$, the presence or absence of a skill at time $t_{2}$ only depends on its presence or absence at a previous time.

Let gain $\gamma_{o s}$ be the probability $P\left(s \in \mathbf{C}_{2} \mid s \notin \mathbf{C}_{1}, o\right)$ that the students presented with learning object $o$ gain the skill $s$ going from the pretest to the posttest, and loss $\lambda_{o s}$ be the probability $P\left(s \notin \mathbf{C}_{2} \mid s \in \mathbf{C}_{1}, o\right)$ that the same students lose it. The conditional probability of state $D$ at the posttest, given state $C$ at the pretest and learning object $o$ turns out to be:

$$
\begin{aligned}
& P\left(\mathbf{C}_{2}=D \mid \mathbf{C}_{1}=C, o\right)=\prod_{s \in S}\left[\lambda_{o s}^{1-w(s, D)}\left(1-\lambda_{o s}\right)^{w(s, D)}\right]^{w(s, C)} \\
& \quad \times\left[\gamma_{o s}^{w(s, D)}\left(1-\gamma_{o s}\right)^{1-w(s, D)}\right]^{1-w(s, C)}
\end{aligned}
$$

where $w(s, C) \in\{0,1\}$ (respectively $w(s, D)$ ) is equal to 1 if skill $s$ belongs to state $C$ (respectively $D$ ).

Via the competency model, a skill multimap associates with each problem a collection of subsets of skills that are necessary and sufficient to solve it. A skill multimap is a triple $(Q, S, \delta)$, where $\delta$ is a mapping from $Q$ to the powerset of $2^{S}$ such that $\delta(q) \neq \varnothing$ for each $q \in Q$, and $C \neq$ $\varnothing$ for each $C \in \delta(q)$ (Doignon, 1994; Doignon \& Falmagne, 1999). Each subset $C$ in $\delta(q)$ represents a competency for $q$. The performance state delineated by $C \subseteq S$ via the competency model is specified by:

$M(C)=\left\{q \in Q: C^{\prime} \subseteq C\right.$ for some $\left.C^{\prime} \in \delta(q)\right\}$,

and the performance structure delineated by the skill multimap $(Q, S, \delta)$ is:

$\mathcal{K}=\{M(C) \mid C \subseteq S\}$.

The performance structure delineated by a skill multimap is a general structure, i.e., it is not necessarily closed under union or intersection (Doignon \& Falmagne, 1999, p. 97).

Let careless error $\alpha_{q}$ be the probability $P\left[\mathbf{R}_{q}=\right.$ $0 \mid q \in M(\mathbf{C})]$ that the students fail problem $q$ given that they possess a competence for it, and lucky guess $\beta_{q}$ be the probability $P\left[\mathbf{R}_{q}=1 \mid q \notin M(\mathbf{C})\right]$ that the same students solve problem $q$ given that they do not posses any competence for it. Therefore, careless error and lucky guess are the error parameters that govern the emission probabilities of response patterns. Assuming responses to the problems are locally independent, given the student's competence state, the conditional probability of response pattern $\mathbf{r}$, given the state $C$, is:

$$
\begin{aligned}
P\left(\mathbf{R}_{t}=\mathbf{r} \mid \mathbf{C}_{t}=C\right)= & \prod_{q=1}^{n}\left[\alpha_{q}^{1-r_{q}}\left(1-\alpha_{q}\right)^{r_{q}}\right]^{v(q, C)} \\
& \times\left[\beta_{q}^{r_{q}}\left(1-\beta_{q}\right)^{1-r_{q}}\right]^{1-v(q, C)}
\end{aligned}
$$

where $t \in\{1,2\}, v(q, C) \in\{0,1\}$ is equal to 1 if problem $q$ is solvable by state $C$, and $r_{q} \in\{0,1\}$ is equal to 1 if problem $q$ is solved.

It should be noted that careless error and lucky guess parameters also characterize the Basic Local Independence Model (BLIM, Doignon \& Falmagne, 1999; Falmagne \& Doignon, 1988) and the Deterministic Inputs Noisy AND gate (DINA) model (Haberman, 1979; Junker \& Sijtsma, 2001; Macready \& Dayton, 1977), that are two approaches often used in knowledge assessment and cognitive diagnosis, respectively. However, there are relevant differences between the GaLoM, the BLIM, and the DINA. Unlike the BLIM, which is developed on the level of the problems without assuming underlying skills, the GaLoM and the DINA assume the existence of discrete skills that underlie the solution of the problems. However, whereas the DINA is a conjunctive model, the presented model is a competency model based on a skill multimap. Moreover, unlike the BLIM and the DINA, the GaLoM allows the assessment of the effect of an educational intervention on the attainment of specific skills.

\section{Parameter estimation of the gain-loss model}

In the GaLoM, an initial probability $\pi_{s}$ is estimated for each skill $s \in S$, a gain parameter $\gamma_{o s}$ and a loss parameter $\lambda_{o s}$ for each learning object $o \in\{1,2, \ldots, m\}$ and each skill $s$, a careless error parameter $\alpha_{q}$ and a lucky guess parameter $\beta_{q}$ for each problem $q \in Q$. Therefore, the total number of parameters to be estimated is $2 n+(1+2 m)|S|$, where $n$ is the number of problems, $m$ is the number of learning objects, and $|S|$ is the cardinality of the set of skills.

Since the GaLoM is essentially a latent class model where the latent classes are the competence states in $C$, maximum likelihood estimates of the parameters can be computed by an application of the ExpectationMaximization (EM) algorithm (Dempster, Laird, \& Rubin, 1977).

Let $\mathbf{X}_{t}$ be a $i \times n$ binary matrix each row of which is the response pattern of student $j \in\{1,2, \ldots, i\}$ to the $n$ problems in $Q$ at time $t \in\{1,2\}$. Each element $x_{t j k} \in\{0,1\}$ in $\mathbf{X}_{t}$ is equal to 1 if student $j$ solved problem $k \in\{1,2, \ldots$, 
$n$ \} at time $t$. The observed data sample is the binary matrix $\mathbf{X}=\left(\mathbf{X}_{1}, \mathbf{X}_{2}\right)$ with dimensions $i \times 2 n$. Let $\boldsymbol{\theta}$ be the vector of all model parameters, and let $\mathbf{o}=\left(o_{1}, o_{2}, \ldots, o_{i}\right)^{\prime}$ be the vector that associates each student to the learning object he was presented with. The observed likelihood of $\mathbf{X}_{1}$ and $\mathbf{X}_{2}$, given the model parameters $\boldsymbol{\theta}$ and the learning objects specified by $\mathbf{0}$, is derived from Eq. (1). Assuming multinomial sampling, it takes on the form:

$l\left(\mathbf{X}_{1}, \mathbf{X}_{2} \mid \theta, \mathbf{o}\right)=\prod_{j=1}^{i}\left[\sum_{C \subseteq S} \sum_{D \subseteq S} P\left(\mathbf{x}_{1 j} \mid C\right) P\left(\mathbf{x}_{2 j} \mid D\right) P\left(D \mid C, o_{j}\right) P(C)\right]$,

where $\mathbf{x}_{1 j}$ (respectively $\mathbf{x}_{2 j}$ ) is the $j$ th row-vector of matrix $\mathbf{X}_{1}$ (respectively $\mathbf{X}_{2}$ ).

The competence states of the students are obviously unknown, but, if there was complete information, each student $j$ would be represented by a quadruple $\left(\mathbf{x}_{1 j}, \mathbf{x}_{2 j}, Y_{1 j}\right.$, $Y_{2 j}$ ), where $\mathbf{x}_{1 j}$ (respectively $\mathbf{x}_{2 j}$ ) is a $1 \times n$ binary vector representing the response pattern of $j$ at time 1 (respectively time 2), and $Y_{1 j}$ (respectively $Y_{2 j}$ ) is the competence state of $j$ at time 1 (respectively time 2). Therefore, the complete data sample would be the quadruple $\left(\mathbf{X}_{1}, \mathbf{X}_{2}, \mathbf{Y}_{1}, \mathbf{Y}_{2}\right)$, and the complete data log-likelihood of the model would turn out to be:

$\ln l\left(\mathbf{X}_{1}, \mathbf{X}_{2}, \mathbf{Y}_{1}, \mathbf{Y}_{2} \mid \theta, \mathbf{o}\right)=\sum_{j=1}^{i} \ln P\left(\mathbf{x}_{1 j}, \mathbf{x}_{2 j}, Y_{1 j}, Y_{2 j} \mid \theta, \mathbf{o}\right)$

where $P\left(\mathbf{x}_{1 j}, \mathbf{x}_{2 j}, Y_{1 j}, Y_{2 j} \mid \boldsymbol{\theta}, \mathbf{o}\right)$ is the joint probability of response patterns $\mathbf{x}_{1 j}$ and $\mathbf{x}_{2 j}$ and of competence states $Y_{1 j}$ and $Y_{2 j}$ given the model parameters $\boldsymbol{\theta}$ and the learning objects specified by $\mathbf{0}$.

In each iteration of the EM algorithm, the conditional expectation of the complete data log-likelihood, given the observed data $\mathbf{X}$ and the model parameters $\boldsymbol{\theta}^{\prime}$ obtained in the previous iteration of the algorithm, is maximized. Indicating with $U\left(\boldsymbol{\theta}, \boldsymbol{\theta}^{\prime}\right)$ this conditional expectation, the following derivation is made:

$$
\begin{aligned}
U\left(\theta, \theta^{\prime}\right)= & E\left[\ln l\left(\mathbf{X}_{1}, \mathbf{X}_{2}, \mathbf{Y}_{1}, \mathbf{Y}_{2} \mid \theta, \mathbf{o}\right) \mid \mathbf{X}, \theta^{\prime}\right] \\
= & E\left[\sum_{j=1}^{i} \ln P\left(\mathbf{x}_{1 j}, \mathbf{x}_{2 j}, Y_{1 j}, Y_{2 j} \mid \theta, o_{j}\right) \mid \mathbf{X}, \theta^{\prime}\right] \\
= & \sum_{j=1}^{i} \sum_{C \subseteq S} \sum_{D \subseteq S}\left[\ln P\left(\mathbf{x}_{1 j}, \mathbf{x}_{2 j}, C, D \mid \theta, o_{j}\right)\right] \\
& \times P\left(C, D \mid \mathbf{x}_{1 j}, \mathbf{x}_{2 j}, o_{j}, \theta^{\prime}\right) \\
= & \sum_{j=1}^{i} \sum_{C \subseteq S} \sum_{D \subseteq S} \ln \left[P\left(\mathbf{x}_{1 j} \mid C\right) P\left(\mathbf{x}_{2 j} \mid D\right) P\left(D \mid C, o_{j}\right) P(C)\right] \\
& \times P\left(C, D \mid \mathbf{x}_{1 j}, \mathbf{x}_{2 j}, o_{j}, \theta^{\prime}\right),
\end{aligned}
$$

where $o_{j}$ is the learning object $o$ student $j$ has been presented with.
The Bayesian posterior probability of competence state at pretest $C$ and posttest $D$, given response pattern at pretest $\mathbf{x}_{1 j}$ and posttest $\mathbf{x}_{2 j}$, learning object $o_{j}$, and previous estimates of model parameters $\boldsymbol{\theta}^{\prime}$, is:

$$
\begin{aligned}
& P\left(C, D \mid \mathbf{x}_{1 j}, \mathbf{x}_{2 j}, o_{j}, \theta^{\prime}\right)=\frac{P\left(\mathbf{x}_{1 j}, \mathbf{x}_{2 j}, C, D \mid o_{j}, \theta^{\prime}\right)}{P\left(\mathbf{x}_{j j}, \mathbf{x}_{2 j} \mid o_{j}, \theta^{\prime}\right)} \\
& =\frac{P\left(\mathbf{x}_{1 j} \mid C, \theta^{\prime}\right) P\left(\mathbf{x}_{2 j} \mid D, \theta^{\prime}\right) P\left(D \mid C, o_{j}, \theta^{\prime}\right) P\left(C \mid \theta^{\prime}\right)}{\sum_{C^{\prime}} \sum_{D^{\prime}} P\left(\mathbf{x}_{1 j} \mid C^{\prime}, \theta^{\prime}\right) P\left(\mathbf{x}_{2 j} \mid D^{\prime}, \theta^{\prime}\right) P\left(D^{\prime} \mid C^{\prime}, o_{j}, \theta^{\prime}\right) P\left(C^{\prime} \mid \theta^{\prime}\right)},
\end{aligned}
$$

For the purposes of brevity, in the sequel $P\left(C, D \mid \mathbf{x}_{1 j}, \mathbf{x}_{2 j}, o_{j}, \theta^{\prime}\right)$ will be indicated with $b_{j C D}$.

The conditional expected log-likelihood $U\left(\boldsymbol{\theta}, \boldsymbol{\theta}^{\prime}\right)$ can be broken up into three functions in the following way:

$U\left(\theta, \theta^{\prime}\right)=U_{1}\left(\theta, \theta^{\prime}\right)+U_{2}\left(\theta, \theta^{\prime}\right)+U_{3}\left(\theta, \theta^{\prime}\right)$,

where $U_{1}\left(\boldsymbol{\theta}, \boldsymbol{\theta}^{\prime}\right), U_{2}\left(\boldsymbol{\theta}, \boldsymbol{\theta}^{\prime}\right)$, and $U_{3}\left(\boldsymbol{\theta}, \boldsymbol{\theta}^{\prime}\right)$ are defined as:

$U_{1}\left(\theta, \theta^{\prime}\right)=\sum_{j=1}^{i} \sum_{C \subseteq S} \sum_{D \subseteq S} b_{j C D} \ln P(C)$,

$U_{2}\left(\theta, \theta^{\prime}\right)=\sum_{j=1}^{i} \sum_{C \subseteq S} \sum_{D \subseteq S} b_{j C D} \ln P\left(D \mid C, o_{j}\right)$

and

$U_{3}\left(\theta, \theta^{\prime}\right)=\sum_{j=1}^{i} \sum_{C \subseteq S} \sum_{D \subseteq S} b_{j C D}\left[\ln P\left(\mathbf{x}_{1 j} \mid C\right)+\ln P\left(\mathbf{x}_{2 j} \mid D\right)\right]$.

Note that $U_{1}$ only depends on the initial probabilities $\pi_{s}$ of the skills, $U_{2}$ only depends on gain $\gamma_{o s}$ and loss $\lambda_{o s}$ parameters, and $U_{3}$ only depends on careless error $\alpha_{q}$ and lucky guess $\beta_{q}$ parameters.

Estimation of the initial probabilities of the skills

In each iteration of the EM algorithm, the function $U_{1}\left(\boldsymbol{\theta}, \boldsymbol{\theta}^{\prime}\right)$ is maximized by setting to zero its first partial derivatives with respect to the parameter $\pi_{s}$ :

$\frac{\partial U_{1}\left(\theta, \theta^{\prime}\right)}{\partial \pi_{s}}=\sum_{j} \sum_{C} b_{j C} \cdot\left[\frac{w(s, C)}{\pi_{s}}-\frac{1-w(s, C)}{1-\pi_{s}}\right]$,

where $b_{j C .}=\sum_{D} b_{j C D}$, and $w(s, C) \in\{0,1\}$ is equal to 1 if skill $s$ belongs to state $C$. By setting the right-hand term of (15) to zero, it follows that:

$$
\begin{aligned}
& \left(1-\pi_{s}\right) \sum_{j} \sum_{C} b_{j C} \cdot w(s, C) \\
& =\pi_{s} \sum_{j} \sum_{C} b_{j C} \cdot[1-w(s, C)] .
\end{aligned}
$$


By solving (16) for $\pi_{s}$, one obtains that:

$\pi_{s}=\frac{\sum_{j} \sum_{C} b_{j C} w(s, C)}{\sum_{j} \sum_{C} b_{j C}}$,

and, given $\sum_{C} b_{j C}=1$, it follows that:

$\pi_{s}=\frac{1}{i} \sum_{j} \sum_{C} b_{j C .} w(s, C)$.

Equation (18) represents the adjustment of the estimates of parameter $\pi_{s}$ in each iteration of the EM algorithm.

Estimation of the gain and loss parameters

In each iteration of the EM algorithm, the function $U_{2}\left(\boldsymbol{\theta}, \boldsymbol{\theta}^{\prime}\right)$ is maximized by setting to zero its first partial derivatives with respect to the parameters $\gamma_{o s}$ and $\lambda_{o s}$. The first partial derivative of $U_{2}\left(\boldsymbol{\theta}, \boldsymbol{\theta}^{\prime}\right)$ with respect to the parameter $\gamma_{\text {os }}$ turns out to be:

$\frac{\partial U_{2}\left(\theta, \theta^{\prime}\right)}{\partial \gamma_{o s}}=\sum_{j \in J_{o}} \sum_{C} \sum_{D} b_{j C D}$

$$
\times\left[\frac{[1-w(s, C)] w(s, D)}{\gamma_{o s}}-\frac{[1-w(s, C)][1-w(s, D)]}{1-\gamma_{o s}}\right],
$$

where $J_{o}=\left\{j: o_{j}=o\right\}$ is the set of the subjects presented with learning object $o$, and $w(s, C) \in\{0,1\}$ (respectively $w$ $(s, D)$ ) is equal to 1 if skill $s$ belongs to state $C$ (respectively $D$ ). By setting the right-hand term of (19) to zero, it follows that:

$$
\begin{aligned}
& \left(1-\gamma_{o s}\right) \sum_{j \in J_{o}} \sum_{C} \sum_{D} b_{j C D}[1-w(s, C)] w(s, D) \\
& =\gamma_{o s} \sum_{j \in J_{o}} \sum_{C} \sum_{D} b_{j C D}[1-w(s, C)][1-w(s, D)] .
\end{aligned}
$$

By solving (20) for $\gamma_{o s}$, one obtains the equation for the adjustment of the estimates of such parameters in each iteration of the EM algorithm:

$\gamma_{o s}=\frac{\sum_{C} \sum_{D} b_{o C D}[1-w(s, C)] w(s, D)}{\sum_{C} b_{o C .}[1-w(s, C)]}$,

where $b_{o C D}=\sum_{j \in J_{o}} b_{j C D}$, and $b_{o C}=\sum_{D} b_{o C D}$.

Following a similar development for the parameters $\lambda_{o s}$, the equation for the adjustment of the estimates of such parameters turns out to be:

$\lambda_{o s}=\frac{\sum_{C} \sum_{D} b_{o C D} w(s, C)[1-w(s, D)]}{\sum_{C} b_{o C} w(s, C)}$.
Estimation of the careless error and lucky guess parameters

In each iteration of the EM algorithm, the function $U_{3}\left(\boldsymbol{\theta}, \boldsymbol{\theta}^{\prime}\right)$ is maximized by setting to zero its first partial derivatives with respect to the parameters $\alpha_{k}$ and $\beta_{k}$. The first partial derivative of $U_{3}\left(\boldsymbol{\theta}, \boldsymbol{\theta}^{\prime}\right)$ with respect to the parameter $\alpha_{k}$ turns out to be:

$$
\begin{aligned}
\frac{\partial U_{3}\left(\theta, \theta^{\prime}\right)}{\partial \alpha_{k}}= & \sum_{j=1}^{i} \sum_{C \subseteq S} \sum_{D \subseteq S} b_{j C D} \\
& \times\left[\frac{\left(1-x_{1 j k}\right) v(k, C)+\left(1-x_{2 j k}\right) v(k, D)}{\alpha_{k}}-\frac{x_{1 j k} v(k, C)+x_{2 j k} v(k, D)}{1-\alpha_{k}}\right],
\end{aligned}
$$

where $v(k, C) \in\{0,1\}$ (respectively $v(k, D))$ is equal to 1 if problem $k$ is solvable by state $C$ (respectively $D$ ). By setting the right-hand term of (23) to zero, it follows that:

$$
\begin{aligned}
& \left(1-\alpha_{k}\right) \sum_{j} \sum_{C} \sum_{D} b_{j C D}\left[\left(1-x_{1 j k}\right) v(k, C)+\left(1-x_{2 j k}\right) v(k, D)\right] \\
& =\alpha_{k} \sum_{j} \sum_{C} \sum_{D} b_{j C D}\left[x_{1 j k} v(k, C)+x_{2 j k} v(k, D)\right.
\end{aligned}
$$

By solving (24) for $\alpha_{k}$, one obtains the equation for the adjustment of the estimates of such parameters in each iteration of the EM algorithm:

$\alpha_{k}=\frac{\sum_{j} \sum_{C}\left[b_{j C .}\left(1-x_{1 j k}\right)+b_{j . C}\left(1-x_{2 j k}\right)\right] v(k, C)}{\sum_{j} \sum_{C}\left(b_{j C .}+b_{j . C}\right) v(k, C)}$,

where $b_{j C}=\sum_{D} b_{j C D}$, and $b_{j . C}=\sum_{D} b_{j D C}$.

Following a similar development for the parameters $\beta_{k}$, the equation for the adjustment of the estimates of such parameters turns out to be:

$\beta_{k}=\frac{\sum_{j} \sum_{C}\left(b_{j C .} x_{1 j k}+b_{j . C} x_{2 j k}\right)[1-v(k, C)]}{\sum_{j} \sum_{C}\left(b_{j C .}+b_{j . C}\right)[1-v(k, C)]}$.

\section{A simulation study}

The capability of the GaLoM to assess the effect of a learning object was tested in a simulation study. The model was assessed with respect to identifiability and goodnessof-recovery, i.e., how well the true model parameters are recovered by the estimation algorithm. Various ratios between the number of problems and underlying skills, effects of the learning object on the skills, and levels of noise in the data were considered. 
Simulation of the data sets

The random data sets were generated according to the GaLoM and 16 conditions. These conditions were produced by considering two ratios between the number of problems and underlying skills, four combinations of learning object effects in gaining and losing the skills, and two levels of noise in the data. With respect to the first, two collections with 10 and 20 problems were generated, and five skills were set to underlie both. Each problem of the two collections has been associated with its competencies according to the skill multimaps represented in Table 1 . The two resulting structures contain 32 states, and they happen to be neither closed under union nor under intersection. These structures were used to generate the data for the conditions with 10 and 20 problems. The learning object was set to highly affect both gain and loss of the skills $\left(\gamma_{\text {true }}\right.$ and $\left.\lambda_{\text {true }}>.66\right)$, to highly affect gain and poorly affect loss $\left(\gamma_{\text {true }}>.66 ; \lambda_{\text {true }}<.33\right)$, to poorly affect gain and highly affect loss $\left(\gamma_{\text {true }}<.33 ; \lambda_{\text {true }}>.66\right)$, to poorly affect both gain and loss $\left(\gamma_{\text {true }}\right.$ and $\left.\lambda_{\text {true }}<.33\right)$. The noise in the data was set to be low $\left(\alpha_{\text {true }}\right.$ and $\left.\beta_{\text {true }}<.1\right)$ in one case, and high $\left(\alpha_{\text {true }}\right.$ and $\left.\beta_{\text {true }}<.3\right)$ in the other case.

The true initial probabilities of the skills were set to be in the interval $[.1, .9]$. This choice was suggested by a preliminary set of simulations where it was observed that, with very high or very low initial probabilities, it becomes difficult to estimate gain and loss probabilities. This occurs because, when the initial probability of a skill is very close to, e.g., 1, the variance of such a skill in the sample is extremely small. Therefore, it is not easy to estimate the probability of gaining the skill in the few students who do not possess it in the pretest. However, it should be noted that, when the initial probability of a skill is very high, estimating gain probability of such skill is negligible. The same thing holds for estimating loss probability of a skill when the initial probability is very low.
The true model parameters were randomly generated according to the aforementioned constraints. Initial probabilities of the skills, gain and loss parameters varied between the two noise conditions whereas they were fixed across the conditions concerning the number of problems and the learning object effect.

For each of the 16 conditions, 500 random data sets were simulated. The number of response patterns (the sample size) was set to 1,000 for each data set.

\section{Testing goodness-of-recovery and identifiability}

The goodness-of-recovery of the GaLoM was tested in each condition. For each of the $500 \times 16=8,000$ random data sets, the model parameters were estimated with the knowledge structure that was used to generate the data. The model identifiability was also tested in each condition. With this aim, one of the 500 simulated data sets was randomly selected for each condition, and the model parameters were estimated 100 times, by randomly varying the initial values between 0 and 1 . The error parameters were randomly generated between 0 and .5 because, when they are higher than .5 , their interpretation as lucky guess and careless error is meaningless.

\section{Results}

Concerning the problem of identifiability, in the conditions with 20 problems parameter estimates do not change by varying their initial values. The condition $\gamma_{\text {true }}$ and $\lambda_{\text {true }}<$ .33 is the only one with ten problems in which the model is identifiable. In the aforementioned conditions, the model is identifiable with both levels of noise in the data. On the contrary, the standard deviations of parameter estimates become pretty high in the conditions $\gamma_{\text {true }}$ and $\lambda_{\text {true }}>.66$, $\gamma_{\text {true }}>.66$ and $\lambda_{\text {true }}<.33, \gamma_{\text {true }}<.33$ and $\lambda_{\text {true }}>.66$ with 10
Table 1 Competencies in collections with 10 and 20 problems

Note: Letters from $a$ to $e$ refer to the five skills

\begin{tabular}{lllllll}
\hline \multirow{2}{*}{ 10-Problems collection } & & \multicolumn{2}{l}{ 20-Problems collection } & & \\
\cline { 1 - 4 } \cline { 5 - 7 } Problem & Competencies & & Problem & Competencies & Problem & Competencies \\
\hline 1 & $\{a\}$ & 1 & $\{a\}$ & 11 & $\{b, c, e\},\{a, b, c\}$ \\
2 & $\{b\}$ & 2 & $\{b\}$ & 12 & $\{a, b, c, d\},\{c, d, e\}$ \\
3 & $\{c\}$ & 3 & $\{c\}$ & 13 & $\{d, e\},\{a, b, d\}$ \\
4 & $\{d\}$ & 4 & $\{d\}$ & 14 & $\{b, c\}$ \\
5 & $\{a, b\},\{e\}$ & 5 & $\{e\}$ & 15 & $\{b, d\}$ \\
6 & $\{b, e\}$ & 6 & $\{a, b\},\{e\}$ & 16 & $\{b, c, d, e\}$ \\
7 & $\{c, e\}$ & 7 & $\{b, e\}$ & 17 & $\{a, c, d, e\}$ \\
8 & $\{c, d\}$ & 8 & $\{c, e\},\{a, b, c\}$ & 18 & $\{a, c, e\}$ \\
9 & $\{a, b, c\},\{b, c, e\}$ & 9 & $\{c, d\}$ & 19 & $\{a, c\}$ \\
10 & $\{a, b, c, d\},\{c, d, e\}$ & 10 & $\{a, e\}$ & 20 & $\{b, c, d\}$ \\
\hline
\end{tabular}


problems and both noise $<.1(S D \mathrm{~s} \leq .10, .13, .14$, respectively) and noise $<.3(S D \mathrm{~s} \leq .25, .11, .22$, respectively). This result suggests that model identifiability depends, among other, on the ratio between the number of problems and that of underlying skills, and on the learning object effect.

The following part concerns the goodness-of-recovery. Only the conditions in which the model is identifiable are presented. For the condition $\gamma_{\text {true }}$ and $\lambda_{\text {true }}<.33$ with ten problems and noise $<.1$, Figure 1 depicts the true parameters $(x$-axis) plotted versus the mean of the parameter estimates (and the related standard errors) reproduced on the 500 simulated data sets ( $y$-axis). The empirical bias of the estimates are negligible for most of the parameters. Standard errors of the estimates are also quite small. Figure 2 depicts the recovery of the model parameters for the same condition with noise $<.3$. It can be seen that empirical biases and standard errors of the estimates are larger than in the condition with noise $<.1$.

Figure 3 depicts the four conditions with 20 problems and noise $<.1$. The empirical bias of the estimates is negligible for all the parameters when $\gamma_{\text {true }}$ and $\lambda_{\text {true }}>.66$, $\gamma_{\text {true }}>.66$ and $\lambda_{\text {true }}<.33$, and $\gamma_{\text {true }}$ and $\lambda_{\text {true }}<.33$, and it is rather high for a few parameters when $\gamma_{\text {true }}<.33$ and $\lambda_{\text {true }}>$ .66. Empirical biases and standard errors of the estimates become larger with noise $<.3$ (see Fig. 4). This result suggests that the model is capable of recovering the true parameters. Not surprisingly, goodness-of-recovery improves when the noise in the data is low.

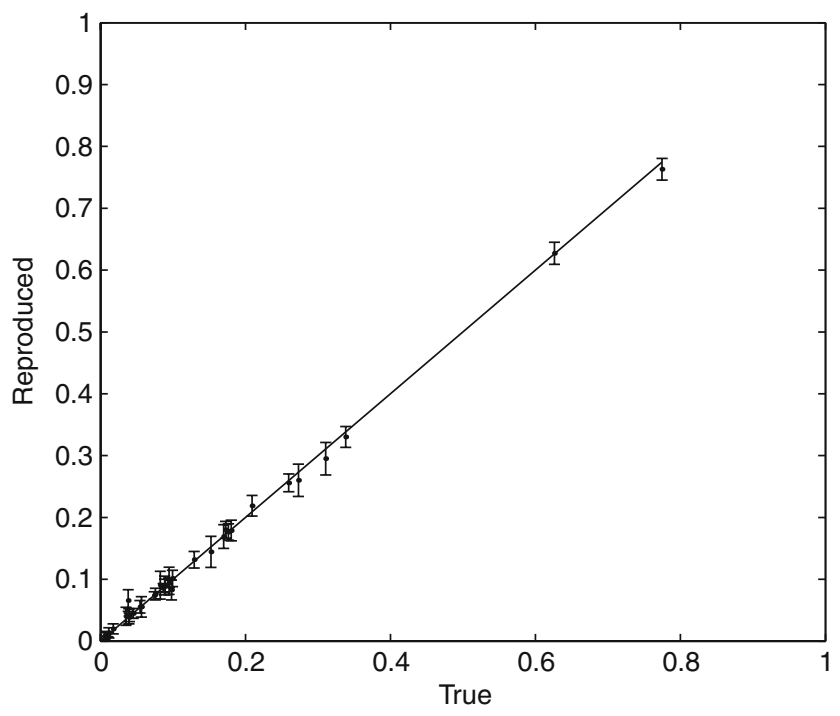

Fig. 1 True parameters ( $x$-axis) versus mean of the parameter estimates (and related standard errors) reproduced on the simulated data sets ( $y$-axis). Condition $\gamma$ and $\lambda<.33$ with ten problems and noise $<.1$. The straight line $x=y$ is added for reference

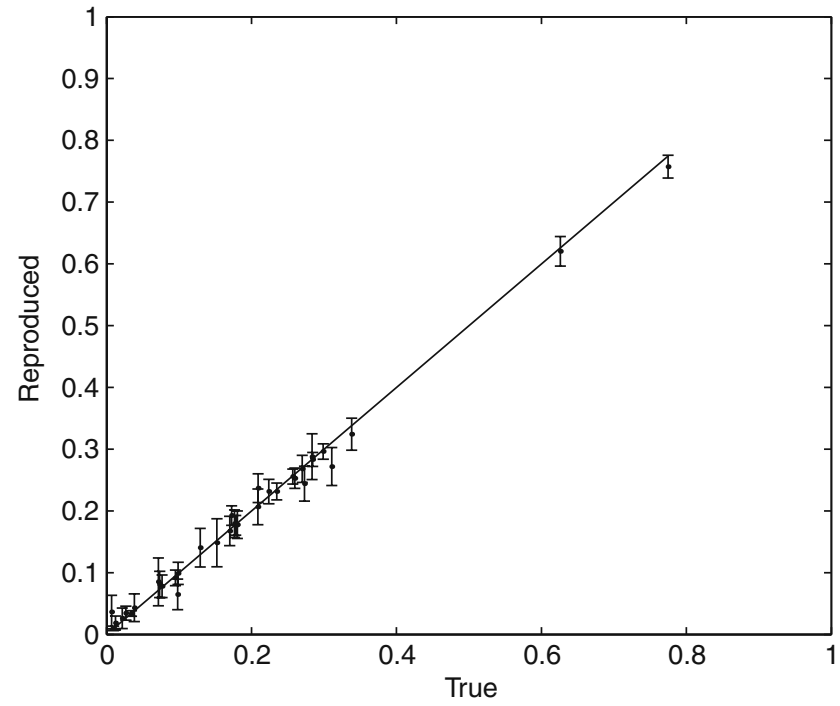

Fig. 2 True parameters ( $x$-axis) versus mean of the parameter estimates (and related standard errors) reproduced on the simulated data sets ( $y$-axis). Condition $\gamma$ and $\lambda<.33$ with ten problems and noise $<.3$. The straight line $x=y$ is added for reference

\section{Discussion}

A probabilistic skill multimap model for assessing learning processes has been presented. The model parameters are the initial probabilities of the skills, the learning object effects on gaining and losing the skills, and the careless error and lucky guess probabilities of the problems.

The model was assessed in a simulation study with respect to identifiability and goodness-of-recovery. Sixteen conditions were produced by considering two ratios between the number of problems and underlying skills, four combinations of learning object effects in gaining and losing the skills, and two levels of noise in the data.

Model identifiability depends on the ratio between the number of problems and underlying skills, and on the learning object effect. It is reasonable to expect that data provide enough information about the skills so that reliable statements about them can be made. When the skills are assessed by means of a small number of problems, compensations between gain and loss parameters and careless error and lucky guess parameters are not excluded. For example, if the gain of a skill is overestimated, the careless error of the problems associated with that skill might be overestimated, whereas if the gain is underestimated, the lucky guess might be overestimated. Such compensations of model parameters might result in multiple solutions for their estimates.

In all cases with no identification problems, the model is able to recover the true parameters. Moreover, recovery of parameters improves when the data have low noise. The noise in the data can be avoided by designing the problems 
Fig. 3 True parameters ( $x$-axis) versus mean of the parameter estimates (and related standard errors) reproduced on the simulated data sets ( $y$-axis). Conditions with 20 problems and noise $<.1$. The straight line $x=y$ is added for reference
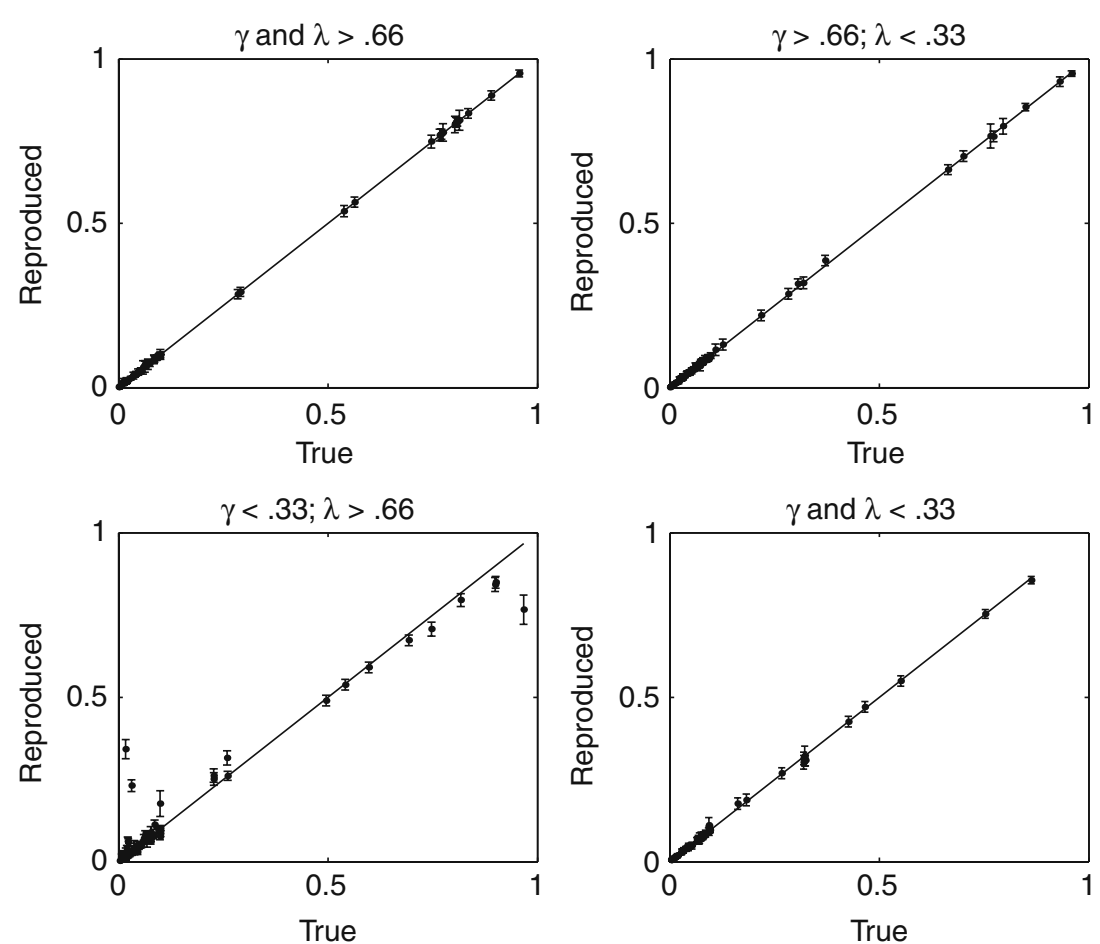

so that their wording does not confuse the students and so that the probability of finding a correct response by chance is small (e.g., open response questions). However, if the noise in the data is high, good recovery of model parameters can still be assured by constraining the error parameter estimates within a certain, reasonably small, interval (Stefanutti \& Robusto, 2009).
A thing to notice is that the condition $\gamma_{\text {true }}$ and $\lambda_{\text {true }}<.33$ behaves well regardless of the number of problems in the collection and the level of noise in the data. Among all conditions with ten problems, this was the only condition in which the model was identifiable (both cases $\alpha, \beta<.1$ and $\alpha, \beta<.3)$. The condition describes a situation in which there is no change between the two assessment steps. It
Fig. 4 True parameters ( $x$-axis) versus mean of the parameter estimates (and related standard errors) reproduced on the simulated data sets ( $y$-axis). Conditions with 20 problems and noise $<.3$. The straight line $x=$ $y$ is added for reference
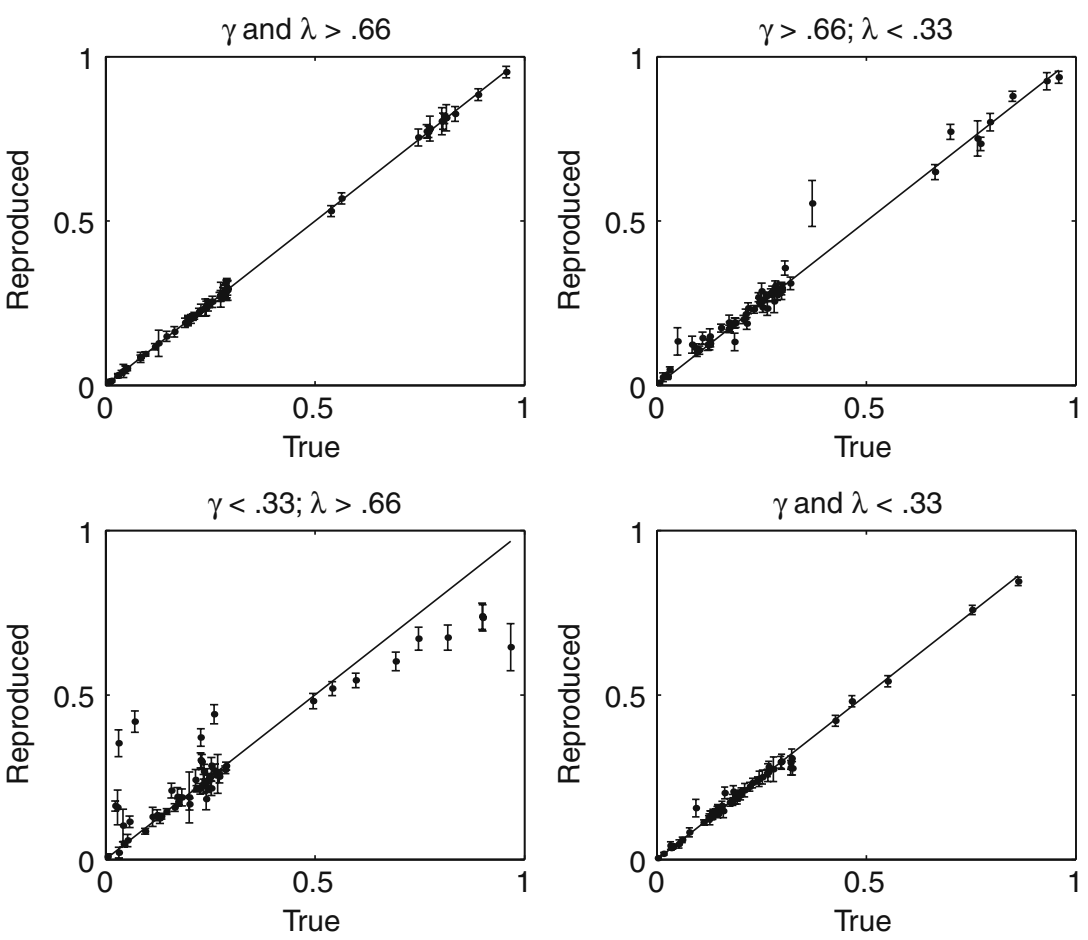
corresponds to the presentation of a learning object that affects neither the attainment nor the loss of skills. Therefore, gain and loss parameters do not affect the competence state of the students between the pretest and the posttest.

The goodness-of-fit of the GaLoM can be assessed by means of Pearson's Chi-square statistic. It should be noted that the approximation to the asymptotic distribution of this statistic lacks accuracy for large and sparse data matrices and cannot be used in practice. This is the case when the observed data sample is too small compared to the theoretical number of distinct binary response patterns $2^{2 \times n}$ (where the 2 in the power is the number of assessment steps and $n$ is the number of problems). In this case, a parametric bootstrap (see, e.g., Langeheine, Pannekoek, \& van de Pol, 1996; von Davier, 1997) can be used to compute the proportion of random data sets whose Chisquare happens to be less than the Chi-square of the observed data set.

The GaLoM assesses the knowledge of the students before the teaching begins and the effectiveness of the learning object in promoting specific learning. The probability of skill $s$ at the end of the learning process as a result of learning object $o$ that has been presented can be easily calculated as $\pi_{s}\left(1-\lambda_{o s}\right)+\left(1-\pi_{s}\right) \gamma_{o s}$. Therefore, the model supports the teacher along two parallel pathways. Along the first pathway the outcome is a detailed skill profile that helps the teacher to assess what the students already know and what they are ready to learn next. The second pathway provides the teacher with substantive information on the effectiveness of the didactic tools he is going to use. As far as a single didactic tool is concerned, the teacher can assess whether it is appropriate (has a low loss probability) for consolidating a skill that the students already possess and whether it is appropriate (has a high gain probability) for facilitating the learning of a new specific skill. By having available a whole collection of didactic tools in the form of exercises and instructions, the teacher can assess which tool is best (has the lowest loss probability) for consolidating a skill and which tool is best (has the highest gain probability) for learning a new skill. The teacher would thus be provided with program evaluative information and with an objective criterion for choosing the didactic tools while a course is taught. This choice could even be personalized according to specific students' skill profiles.

The aspects discussed so far concern the use of the model at a classroom level. However, once the model has been estimated and validated on a suitable sample of students, it can be used to obtain diagnostic information at the student level. Posterior estimates of the parameters can be obtained for each student. Moreover, it is possible to evaluate a single students' proficiency through a detailed skill profile. Such a profile can be obtained both before and after a specific didactic intervention takes place. The latent set of skills possessed by a student can be recovered by taking as the competence state of the student the one that has the highest posterior probability, given the observed response pattern and the parameters of the model. The teacher would thus be provided with formative information for choosing the best educational interventions according to the specific needs and weaknesses of each student.

Careless error and lucky guess parameters provide information about the goodness of the relationship between latent competence states and observable responses. In fitting a knowledge structure to an empirical data set, it is desirable that their estimates remain reasonably small. In fact, high error parameters are difficult to interpret. A possible interpretation is that the data are highly noisy. Problems with a high careless error rate are failed by students who are able to solve them. Problems with a high lucky guess rate are solved by students who are not able to solve them. Another interpretation is that the skill multimap and the corresponding knowledge structure are not appropriate, even if the model fit is acceptable or good. Problems with high careless error could require more skills than expected, whereas those with high lucky guess could be associated with more skills than needed, or could be solved by solution strategies that have not been mapped.

Constraining the error estimates within a certain interval helps to distinguish between noise of the data and misspecifications of the skill multimap. This is particularly useful in practical applications in which there is not much theory about the knowledge structure on a given set of problems and, therefore, the first aim is to establish which of the models at hand is a better approximation of the "true" knowledge structure underlying the data. In particular, when having available a collection of knowledge structures, the introduction of a reasonably small upper bound to the error parameters helps to identify the knowledge structure that best approximates the "true" one. In fact, as the upper bound gets lower, the likelihood of the best structure is expected to decrease much slower than that of the other structures (Stefanutti \& Robusto, 2009). Once the best knowledge structure has been identified, it is possible that for this structure some of the $\alpha$ and $\beta$ parameters lie exactly on the upper bound. In this case, the upper bound could be gradually elevated up to a reasonable value and model parameters reestimated. If at the end of this process there are still some problems whose error parameters are on the upper bound, this could be a sign of too much noise in the data concerning such problems.

The number of students that is required for the model to be applicable in real educational settings is a function of several elements such as the number of problems the students are presented with, the number of skills that are hypothesized to underlie the problems, and the frequency 
with which the different response patterns occur in the population.

A comparison can be made between the presented model and the Stochastic Learning Path (SLP) model (Falmagne, 1993; see also Falmagne, 1989, 1996; Falmagne \& Lakshminarayan, 1994; Lakshminarayan \& Gilson, 1998), that is an approach proposed in knowledge space theory framework for describing the progress of students learning a particular field. A first difference between the two models is that the SLP focuses on the solution behaviour on the level of the problems, whereas the GaLoM focuses on the discrete skills that underlie the solution of the problems. Another difference is that the SLP assesses learning by analyzing the progress of students in a collection of learning paths (i.e., chains of performance states ordered by inclusion and containing $\varnothing$ and $Q$ ). Differently, in the GaLoM the particular learning paths followed by the students in between the two assessment steps are not taken into account. Another difference is that, whereas in the GaLoM it is possible to comparatively assess different educational interventions at once, an extension of the SLP to allow this type of comparison is not trivial. Finally, in the GaLoM there is the possibility of modeling a certain kind of forgetting through the loss parameters, whereas the SLP is only suitable for modeling monotonic learning processes.

The model in the current specification assumes the skills to be stochastically independent. Some pathways for specifying dependencies among the skills include loglinear models (see, e.g., Maris, 1999) or Bayesian networks (see, e.g., Mislevy \& Gitomer, 1996). The model applies to learning processes with two assessment steps. Its extension to any number of assessment steps qualifies it as a hidden Markov model. Another possible extension might take into account whether a competence state contains or not the prerequisites for understanding a given learning object.

\section{References}

Albert, D., \& Held, T. (1999). Component-based knowledge spaces in problem solving and inductive reasoning. In D. Albert \& J. Lukas (Eds.), Knowledge spaces: Theories, empirical research, and applications (pp. 15-40). Mahwah: Lawrence Erlbaum Associates.

Almond, R. G., DiBello, L. V., Moulder, B., \& Zapata-Rivera, J.-D. (2007). Modeling diagnostic assessments with Bayesian networks. Journal of Educational Measurement, 44, 341-359. doi:10.1111/j.1745-3984.2007.00043.x

Conlan, O., Hockemeyer, C., Wade, V., \& Albert, D. (2002). Metadata driven approaches to facilitate adaptivity in personalized eLearning systems. The Journal of Information and Systems in Education, 1, 38-44.

Dempster, A. P., Laird, N. M., \& Rubin, D. B. (1977). Maximum likelihood from incomplete data via the EM algorithm. Journal of the Royal Statistical Society: Series B: Methodological, 39, 1-38.

DiBello, L. V., \& Stout, W. (2007). Guest editors' introduction and overview: IRT-based cognitive diagnostic models and related methods. Journal of Educational Measurement, 44, 285-291. doi:10.1111/j.1745-3984.2007.00039.x

Doignon, J.-P. (1994). Knowledge spaces and skill assignments. In G. H. Fischer \& D. Laming (Eds.), Contributions to mathematical psychology, psychometrics, and methodology (pp. 111-121). New York: Springer-Verlag.

Doignon, J.-P., \& Falmagne, J.-C. (1985). Spaces for the assessment of knowledge. International Journal of ManMachine Studies, 23, 175-196. doi:10.1016/S0020-7373(85) 80031-6

Doignon, J.-P., \& Falmagne, J.-C. (1999). Knowledge spaces. Berlin: Springer-Verlag.

Falmagne, J.-C. (1989). A latent trait theory via stochastic learning theory for a knowledge space. Psychometrika, 54, 283-303. doi:10.1007/BF02294521

Falmagne, J.-C. (1993). Stochastic learning paths in a knowledge structure. Journal of Mathematical Psychology, 37, 489-512. doi:10.1006/jmps.1993.1031

Falmagne, J.-C. (1996). Errata to SLP. Journal of Mathematical Psychology, 40, 169-174.

Falmagne, J.-C., \& Doignon, J.-P. (1988). A class of stochastic procedures for the assessment of knowledge. The British Journal of Mathematical and Statistical Psychology, 41, 1-23.

Falmagne, J.-C., Doignon, J.-P., Koppen, M., Villano, M., \& Johannesen, L. (1990). Introduction to knowledge spaces: How to build, test, and search them. Psychological Review, 97, 201224. doi:10.1037/0033-295X.97.2.201

Falmagne, J.-C., \& Lakshminarayan, K. (1994). Stochastic learning paths - Estimation and simulation. In G. H. Fischer \& D. Laming (Eds.), Contributions to mathematical psychology, psychometrics, and methodology (pp. 91-110). New York: Springer-Verlag.

Gierl, M. J. (2007). Making diagnostic inferences about cognitive attributes using the rule-space model and attribute hierarchy method. Journal of Educational Measurement, 44, 325-340. doi:10.1111/j.1745-3984.2007.00042.x

Haberman, S. (1979). Qualitative data analysis (Vols. 1, 2). New York: Academic.

Heller, J., \& Repitsch, C. (2008). Distributed skill functions and the meshing of knowledge structures. Journal of Mathematical Psychology, 52, 147-157. doi:10.1016/j.jmp.2008.01.003

Heller, J., Steiner, C., Hockemeyer, C., \& Albert, D. (2006). Competence-based knowledge structures for personalised learning. International Journal on E-Learning, 5, 75-88.

Hockemeyer, C. (2003). Competence based adaptive e-learning in dynamic domains. In F. W. Hesse \& Y. Tamura (Eds.), The joint workshop of cognition and learning through media-communication for advanced e-learning (JWCL) (pp. 79-82). Berlin.

Junker, B. W., \& Sijtsma, K. (2001). Cognitive assessment models with few assumptions, and connections with nonparametric item response theory. Applied Psychological Measurement, 25, 258272. doi:10.1177/01466210122032064

Korossy, K. (1999). Modeling knowledge as competence and performance. In D. Albert \& J. Lukas (Eds.), Knowledge spaces: Theories, empirical research, and applications (pp. 103-132). Mahwah: Lawrence Erlbaum Associates.

Lakshminarayan, K., \& Gilson, F. (1998). An application of a stochastic knowledge structure model. In C. E. Dowling, F. S. Roberts, \& P. Theuns (Eds.), Recent progress in mathematical psychology, scientific psychology series (pp. 155-172). Mahwah: Lawrence Erlbaum Associates.

Langeheine, R., Pannekoek, J., \& van de Pol, F. (1996). Bootstrapping goodness-of-fit measures in categorical data analysis. Sociological Methods and Research, 24, 492-516. doi:10.1177/ 0049124196024004004

Lukas, J., \& Albert, D. (1999). Knowledge structures: What they are and how they can be used in cognitive psychology, test theory, 
and the design of learning environments. In D. Albert \& J. Lukas (Eds.), Knowledge spaces: Theories, empirical research, and applications (pp. 3-12). Mahwah: Lawrence Erlbaum Associates.

Macready, G. B., \& Dayton, C. M. (1977). The use of probabilistic models in the assessment of mastery. Journal of Educational and Behavioral Statistics, 2, 99-120. doi:10.3102/10769986002002099

Maris, E. (1999). Estimating multiple classification latent class models. Psychometrika, 64, 187-212. doi:10.1007/BF02294535

Mislevy, R. J., \& Gitomer, D. H. (1996). The role of probability-based inference in an intelligent tutoring system. User Modeling and User-Adapted Interaction, 5, 253-282. doi:10.1007/BF01126112

Robusto, E., Stefanutti, L., \& Anselmi, P. (2010). The Gain-Loss Model: A probabilistic skill multimap model for assessing learning processes. Journal of Educational Measurement, 47, 373-394.

Roussos, L. A., Templin, J. L., \& Henson, R. A. (2007). Skills diagnosis using IRT-based latent class models. Journal of Educational Measurement, 44, 293-311. doi:10.1111/j.17453984.2007.00040.x
Schrepp, M. (1999). An empirical test of a process model for letter series completion problems. In D. Albert \& J. Lukas (Eds.), Knowledge spaces: Theories, empirical research, and applications (pp. 133-153). Mahwah: Lawrence Erlbaum Associates.

Schrepp, M., Held, T., \& Albert, D. (1999). Component-based construction of surmise relations for chess problems. In D. Albert \& J. Lukas (Eds.), Knowledge spaces: Theories, empirical research, and applications (pp. 41-66). Mahwah: Lawrence Erlbaum Associates.

Stefanutti, L., \& Robusto, E. (2009). Recovering a probabilistic knowledge structure by constraining its parameter space. Psychometrika, 74, 83-96. doi:10.1007/s11336-008-9095-7

Stout, W. (2007). Skills diagnosis using IRT-based continuous latent trait models. Journal of Educational Measurement, 44, 313-324. doi:10.1111/j.1745-3984.2007.00041.x

von Davier, M. (1997). Bootstrapping goodness-of-fit statistics for sparse categorical data: results of a Monte Carlo study. Methods of Psychological Research, 2, 29-48. 